\title{
Premier rapport sur la prolifération de marées jaunes ichthyotoxiques à Prymnesium parvum Carter (Haptophyceae) dans le lac hypereutrophe Oued Mellah (Maroc) First report on the occurrence of ichthyotoxic yellow tides of Prymnesium parvum Carter (Haptophyceae) in Oued Mellah hypertrophic lake (Morocco)
}

\author{
B. Sabour, M. Loudiki, B. Oudra, S. Oubraim, B. Fawzi, S. Fadlaoui et M. Chlaida
}

Volume 15, numéro 1, 2002

URI : https://id.erudit.org/iderudit/705456ar

DOI : https://doi.org/10.7202/705456ar

\section{Aller au sommaire du numéro}

\section{Éditeur(s)}

Université du Québec - INRS-Eau, Terre et Environnement (INRS-ETE)

\section{ISSN}

0992-7158 (imprimé)

1718-8598 (numérique)

\section{Découvrir la revue}

Citer cet article

Sabour, B., Loudiki, M., Oudra, B., Oubraim, S., Fawzi, B., Fadlaoui, S. \& Chlaida, M. (2002). Premier rapport sur la prolifération de marées jaunes ichthyotoxiques à Prymnesium parvum Carter (Haptophyceae) dans le lac hypereutrophe Oued Mellah (Maroc). Revue des sciences de l'eau / Journal of Water Science, 15(1), 327-342. https://doi.org/10.7202/705456ar

\section{Résumé de l'article}

Les algues Prymnesiophyceae ou Haptophyceae (Chrysophyta) renferment des espèces capables de pullulations spectaculaires nommées " marées jaunes ". Parmi les Haptophycées, les plus communes sont : Prymnesium parvum, Prymnesium patelliferum et Chrysochromulina polylepis. Ces microalgues ont été souvent incriminées dans des cas de mortalité aiguë de poissons et d'invertébrés aquatiques. P. parvum est connue depuis cent ans comme responsable de blooms toxiques ayant entraîné des mortalités catastrophiques de poissons dans diverses régions du monde.

$\mathrm{Au}$ Maroc, ces blooms à Prymnesiophyceae n'ont, jusqu'à présent, jamais été signalés. Nos recherches sur les successions phytoplanctoniques du lac hypereutrophe et saumâtre de Oued Mellah $\left(33^{\circ} 30^{\prime} \mathrm{N}-07^{\circ} 20^{\prime} \mathrm{W}\right)$, ont mis en évidence la prolifération périodique en automne de l'Haptophycée P. parvum occasionnant des blooms spectaculaires. Les densités cellulaires de P. parvum, espèce dominante, atteignent des maxima de $148 \cdot 10^{6}$ cellules $\mathrm{L}^{-1}$ en 1998 et $169 \cdot 10^{6}$ cellules $\cdot \mathrm{L}^{-1}$ en 1999 , représentant 34 à $80 \%$ de la biomasse totale. Ces " marées jaunes " coïncident régulièrement avec des mortalités catastrophiques de poissons et d'invertébrés aquatiques dans le lac laissant présager une toxicité aiguë de cette souche de microalgue.

Dans ce travail sont présentés les premiers résultats concernant la dynamique interannuelle des blooms à P. parvum et l'influence des conditions physicochimiques particulières des eaux du lac sur leur développement est discutée. 


\title{
Premier rapport sur la prolifération de marées jaunes ichthyotoxiques à Prymnesium parvum Carter (Haptophyceae) dans le lac hypereutrophe Oued Mellah (Maroc)
}

\author{
First report on the occurrence of ichthyotoxic yellow \\ tides of Prymnesium parvum Carter (Haptophyceae) \\ in Oued Mellah hypertrophic lake (Morocco)
}

\author{
B. SABOUR ${ }^{1}$, M. LOUDIKI $^{2}{ }^{\star}$, B. OUDRA ${ }^{2}$, S. OUBRAIM ${ }^{3}$, B. FAWZI $^{3}$, \\ S. FADLAOUI ${ }^{3}$, M. CHLAIDA $^{3}$
}

\section{SUMMARY}

\begin{abstract}
The Prymnesiophyceae or Haptophyceae (Chrysophyta) contain species-forming spectacular blooms named yellow tides. Among Haptophyceae, Prymnesium parvum, Prymnesium patelliferum and Chrysochromulina polylepis are the most common. These microalgae were often involved in acute fish and aquatic invertebrates mortality cases. $P$. parvum, known since hundred years as being responsible for toxic blooms, has induced catastrophic fish mortality in various areas throughout the world.
\end{abstract}

In Morocco, blooms of Prymnesiophyceae have never been reported up till now. Studies on phytoplankton successions in the hypertrophic brackish Oued Mellah lake $\left(33^{\circ} 30^{\prime} \mathrm{N}-07^{\circ} 20^{\prime} \mathrm{W}\right)$ show a periodic autumnal proliferation of $P$. parvum. This species, which cellular densities reach a maxima of $148 \cdot 10^{6}$ cells $\mathrm{L}^{-1}$ in 1998 and $169 \cdot 10^{6}$ cells $\mathrm{L}^{-1}$ in 1999 , dominates the other phytoplanctonic species with 34 to $80 \%$ of the total biomass. The yellow tides coincide regularly with dramatic fish and aquatic invertebrates mortality in the lake leading to the prediction of an acute toxicity due to this microalgae.

The principal aim of this study was to present the first results concerning the interannual dynamies of $P$. parvum blooms and to discuss the influence of the particular physical and chemical water characteristics on their development.

Key-words: blooms, Prymnesium parvum, dynamics, ichthyotoxicity, Morocco.

1. Faculté des sciences et techniques Mohammadia, Département de biologie, Mohammadia, Maroc.

2. Faculté des sciences Semlalia, Département de biologie, Laboratoire d'algologie, BP 2390,40000 Marrakech, Maroc.

3. Faculté des sciences Ben M'sik, Département de biologie, Laboratoire d'écologie et d'environnement BP 7955 Sidi Othmane, Casablanca, Maroc.

* Correspondance. E-mail : loudiki@ucam.ac.ma

Les commentaires seront reçus jusqu'au 31 mars 2003. 


\section{RÉSUMÉ}

Les algues Prymnesiophyceae ou Haptophyceae (Chrysophyta) renferment des espèces capables de pullulations spectaculaires nommées « marées jaunes $»$. Parmi les Haptophycées, les plus communes sont : Prymnesium parvum, Prymnesium patelliferum et Chrysochromulina polylepis. Ces microalgues ont été souvent incriminées dans des cas de mortalité aiguë de poissons et d'invertébrés aquatiques. $P$. parvum est connue depuis cent ans comme responsable de blooms toxiques ayant entraîné des mortalités catastrophiques de poissons dans diverses régions du monde.

Au Maroc, ces blooms à Prymnesiophyceae n'ont, jusqu'à présent, jamais été signalés. Nos recherches sur les successions phytoplanctoniques du lac hypereutrophe et saumâtre de Oued Mellah $\left(33^{\circ} 30^{\prime} \mathbf{N}-07^{\circ} 20^{\prime} W\right)$, ont mis en évidence la prolifération périodique en automne de l'Haptophycée $P$. parvum occasionnant des blooms spectaculaires. Les densités cellulaires de $\boldsymbol{P}$. parvum, espèce dominante, atteignent des maxima de $148 \cdot 10^{6}$ cellules $^{-1} L^{-1}$ en 1998 et $169 \cdot 10^{6}$ cellules $\cdot \mathrm{L}^{-1}$ en 1999 , représentant 34 à $80 \%$ de la biomasse totale. Ces « marées jaunes » coöncident régulièrement avec des mortalités catastrophiques de poissons et d'invertébrés aquatiques dans le lac laissant présager une toxicité aiguë de cette souche de microalgue.

Dans ce travail sont présentés les premiers résultats concernant la dynamique interannuelle des blooms à $\boldsymbol{P}$. parvum et l'influence des conditions physicochimiques particulières des eaux du lac sur leur développement est discutée.

Mots clés : Prolifération, Prymnesium parvum, dynamique, ichthyotoxicité, Maroc.

\section{1 - INTRODUCTION}

Durant le siècle précédant, et plus particulièrement au cours sa dernière décade, des espèces appartenant au genre Prymnesium (Haptophyceae) ont été incriminées dans des cas de mortalités de poissons et d'autres animaux aquatiques dans plusieurs régions du monde (BELL, 1980 ; EDVARDSEN et PAASCHE, 1998 ; FARROW, 1969 ; GUO et al., 1996 ; KONONEN et al., 1993 ; LINDHOLM et al., 1999 ; MOESTRUP, 1994 ; OTTERSTRØM et STEEMANN NIELSON, 1940). Prymnesium parvum, algue unicellulaire biflagellée, fréquente surtout dans les plans d'eau saumâtre, est l'espèce la plus commune du genre. Cette microalgue euryhaline se rencontre généralement dans les eaux côtières (mer Baltique par exemple), les estuaires, les lacs et les étangs saumâtres hautement eutrophisés où elle trouve sa niche écologique (CHRETIENNOT-DINET, 1990 ; PREMAZZI et VOLTERRA, 1993). Les premiers rapports sur l'implication de cette espèce dans les mortalités de poisson ont trait au lac Workum en Hollande (LIEBERT et DEERNS, 1920) et à des eaux saumâtres estuariennes en Angleterre (CARTER, 1937). Plus tard, cette espèce a été associée à des épisodes d'ichthyotoxicité en Europe du Nord et au Moyen-Orient avec comme conséquence des événements de toxicité particulièrement sérieux dans des fermes piscicoles en Finlande, Norvège, Danemark, Angleterre, Bulgarie, Hol lande, Italie, Chine... (PREMAZZl et VOLTERRA, 1993). Au Maroc, les études réalisées sur les lacs naturels et retenues de barrages montrent qu'ils constituent le 
siège de formation de blooms phytoplanctoniques formés essentiellement par les cyanobactéries (DERRAZ, 1995 ; LOUDIKI et al., 2000 ; OUDRA et al., 1998).

L'étude hydrobiologique entamée depuis 1997 sur le lac du barrage Oued Mellah, montre qu'en plus des blooms successifs à cyanobactéries toxiques (SABOUR et al., 2000), le lac est le siège du développement excessif de l'haptophycée $P$. parvum qui forme des blooms spectaculaires en automne (1998 et 1999). Ces blooms coïncident avec des mortalités aiguës de poissons et d'invertébrés aquatiques. À notre connaissance, $P$. parvum est inventoriée pour la première fois dans les plans d'eau du Maroc. Sa présence et sa prolifération dans le lac témoignent de conditions écologiques particulières. L'objectif de ce travail est de présenter et de discuter les premières données concernant la dynamique interannuelle des blooms à $P$. parvum survenus en 1998 et 1999 , d'analyser l'influence des conditions physicochimiques particulières des eaux du lac sur son développement et d'évaluer les conséquences sur les autres composantes biotiques du milieu.

\section{2 - MATÉRIEL ET MÉTHODES}

La retenue du barrage Oued Mellah se situe sur le cours d'eau nommé Oued Mellah à $30 \mathrm{~km}$ de son embouchure sur l'océan atlantique et à $25 \mathrm{~km}$ au nord-est de Casablanca (figure 1). Les caractéristiques de ce lac sont données dans le tableau 1.

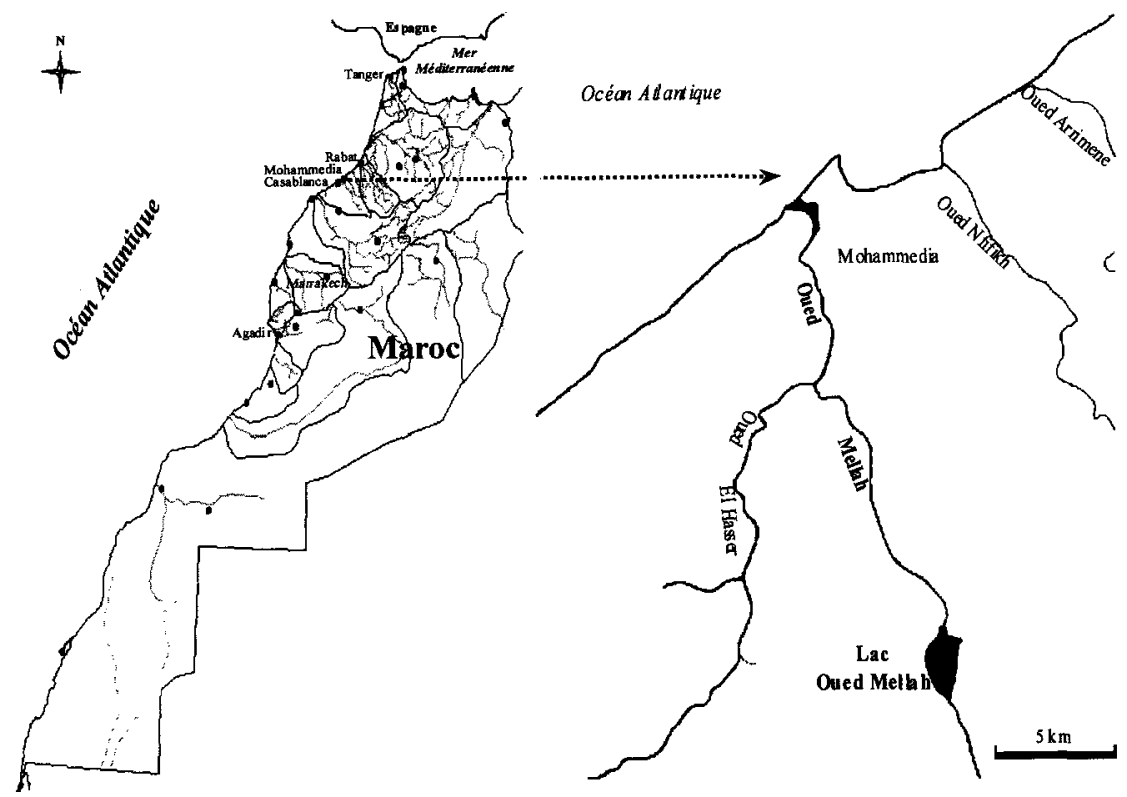

Figure 1 Situation géographique du lac Oued Mellah.

Geographic situation of Oued Mellah lake. 
Tableau 1 Caractéristiques générales du lac du barrage Oued Mellah.

Table 1 General characteristics of Oued Mellah lake reservoir.

\begin{tabular}{l|l} 
Lac de barrage & Oued Mellah \\
Date de construction & $1928-1931$ \\
Situation géographique & $33^{\circ} 30^{\prime} \mathrm{N}-07^{\circ} 20^{\prime} \mathrm{W}, 25 \mathrm{~km}$ au nord de Casablanca \\
Bassin versant & Côtiers atlantiques de Casablanca \\
Cours d'eau & Oued Mellah \\
Capacité totale & $18 \mathrm{Mm}^{3}$ \\
Capacité utile & $8,8 \mathrm{Mm}^{3}$ \\
Surface & $250 \mathrm{ha}$ \\
Cote normale & $101,75 \mathrm{NGM}$ \\
Surface du bassin versant & $1800 \mathrm{~km}^{2}$ \\
Profondeur moyenne & $4 \mathrm{~m}$ \\
Profandeur maximale & $9 \mathrm{~m}$ \\
Géologie des versants & Schistes primaires surmontés par la dale grésomaghrébienne \\
& avec des affleurements du trias (argile salifère et basalte altéré) \\
Bioclimat & Thermoméditerranéen semi-aride à hiver chaud \\
Utilisation & Production d'eau domestique, irrigation, électricité, pêche \\
et recréation \\
Intérêt écologique & Site d'intérêt biologique et écologique (SIBE nH8)
\end{tabular}

Les échantillons d'eau ont été prélevés entre $10 \mathrm{~h}$ et midi à différentes profondeurs $(0,1,2,4 \mathrm{~m}$ et le fond) au niveau du secteur le plus profond du lac $(7-9 \mathrm{~m})$ à l'aide d'une bouteille conçue selon le modèle Van Dorn (capacité 2,5 litres). Les analyses physicochimiques des eaux ont été réalisées selon les méthodes AFNOR (1994) et RODIER (1984). L'extraction de la chlorophylle a, retenue après filtration d'un volume de 50 à $1000 \mathrm{~mL}$ (selon la densité en phytoplancton) sur des filtres Wathman GF/C, a été réalisée avec l'acétone à $90 \%$. Après 24 heures d'extraction à l'obscurité et au froid $\left(4^{\circ} \mathrm{C}\right)$, les extraits ont été centrifugés et les densités optiques mesurées à $665 \mathrm{~nm}$ avant et après acidification par $\mathrm{HCl} 1 \mathrm{~N}$. Les équations de LORENZEN (1967) ont été utilisées pour le calcul des concentrations en chlorophylle a. Pour l'analyse qualitative du phytoplancton et l'établissement de l'inventaire des taxons, des traits verticaux et horizontaux ont été réalisés à l'aide d'un filet à plancton de $40 \mu \mathrm{m}$ de vide de maille et les concentrés d'algues récoltés ont servi à l'identification au microscope optique. La détermination des espèces de petite taille non retenues par le filet a été effectuée au microscope après concentration des échantillons du phytoplancton prélevés à l'ajde de la bouteille par sédimentation. L'échantillonnage quantitatif du phytoplancton a été effectué à l'aide de la bouteille aux profondeurs retenues selon une fréquence mensuelle sauf en période de blooms à $P$. parvum où les prélèvements en surface du lac ont été hebdomadaires pour le suivi rapproché de la cinétique de croissance. Pour chaque profondeur, un sous-échantillon de $500 \mathrm{~mL}$, destiné au comptage du phytoplancton, était immédiatement fixé au formol neutre ou lugol à $5 \%$. Le comptage du phytoplancton a été effectué au microscope inversé selon la méthode d'UTERMÖHL (1958). La conversion des résultats en biomasse exprimée en poids frais a été faite en déterminant le biovolume de chaque espèce et en admettant que la densité algale est voisine de celle de l'eau $\left(10^{6} \cdot \mathrm{m}^{3} \cdot \mathrm{L}^{-1}=1 \mu \mathrm{g}\right.$ de poids frais $\left.\cdot \mathrm{L}^{-1}\right)$ (CAPBLANCQ, 1982). Le taux de croissance $(\mu)$ de Prymnesium parvum a été calculé à 
partir de deux évaluations successives de la densité de l'espèce D1 et D2 respectivement aux temps T1 et T2 $: \mu=\operatorname{Ln}(\mathrm{D} 2 / \mathrm{D} 1) \times(1 / \mathrm{T} 2-\mathrm{T} 1)$. En période de blooms à $P$. parvum des observations ont été notées au cours des prospections hebdomadaires des zones de berges du lac pour l'évaluation qualitative (liste des espèces) des mortalités de poissons et de macro-invertébrés. Des spécimens de poissons intoxiqués encore vivants ont été récoltés pour l'étude histopathologique (branchies, foie, intestins) réalisée aimablement par Mme M. El-Andaloussi Dadi du Laboratoire d'histopathologie Tensift (Marrakech).

\section{3 - RÉSULTATS}

\subsection{Hydrochimie}

L'étude de la qualité physicochimique des eaux du lac du barrage Oued Mellah durant les années 1997, 1998 et 1999 montre qu'il s'agit d'un lac non stratifié (écart thermique maximal surface-fond : $3,5^{\circ} \mathrm{C}$ ), saumâtre (moyenne interannuelle : $6,4 \%$ ) et hypereutrophe (moyenne interannuelle de la chlorophylle a : $\left.75,63 \mu \mathrm{g} \cdot \mathrm{L}^{-1}\right)$.

Les données des principaux paramètres physicochimiques des eaux de surface du lac Oued Mellah en périodes de prolifération maximale de $P$, parvum (novembre-décembre 1998 et septembre-octobre 1999) sont présentées dans le tableau 2.

Tableau 2 Quelques paramètres physicochimiques mesurés en périodes de blooms à Prymnesium parvum.

Table 2 Some physical and chemical parameters measured in Prymnesium parvum bloom periods.

\begin{tabular}{|c|c|c|c|c|}
\hline & \multicolumn{2}{|c|}{1998} & \multicolumn{2}{|c|}{1999} \\
\hline & $\begin{array}{l}\text { Valeurs } \\
\text { extrêmes }\end{array}$ & Moyenne & $\begin{array}{l}\text { Valeurs } \\
\text { extrêmes }\end{array}$ & Moyenne \\
\hline Lumière incidente $\left(\mu \mathrm{mol} \cdot \mathrm{m}^{-2} \cdot \mathrm{s}^{-1}\right)$ & $1140-1491$ & 1238 & $509-1807$ & 1304 \\
\hline Zs (m) & $0,28-0,45$ & 0,36 & $0,28-0,36$ & 0,34 \\
\hline $\mathrm{Ze}(\mathrm{m})$ & $0,73-1,17$ & 0,94 & $0,73-0,94$ & 0,88 \\
\hline Température eau $\left({ }^{\circ} \mathrm{C}\right)$ & $15-23,5$ & 18,5 & $23,5-24$ & 23,6 \\
\hline $\mathrm{pH}$ & $8,3-8,9$ & 8,7 & $8,4-9,0$ & 8,7 \\
\hline Salinité (\%o) & $8,61-8,93$ & 8,8 & $12,25-12,64$ & 12,4 \\
\hline Conductivité $\left(\mathrm{ms} \cdot \mathrm{cm}^{-1}\right)$ & $4,25-4,63$ & 4,43 & $4,73-5,04$ & 4,89 \\
\hline Oxygène dissous (\%) & $98,5-127,6$ & 113,1 & $58,1-121,9$ & 90,0 \\
\hline Orthophosphates $\left(\mu \mathrm{g} \cdot \mathrm{L}^{-1}\right)$ & \multicolumn{4}{|c|}{$<$ limite de détection de la méthode $\left(5 \mu \mathrm{g} \cdot \mathrm{L}^{-1}\right)$} \\
\hline Phosphore total $\left(\mu \mathrm{g} \cdot \mathrm{L}^{-1}\right)$ & $7,7-14,7$ & 11,2 & $18,4-21,7$ & 20,1 \\
\hline Nitrates $\left(\mu \mathrm{g} \cdot \mathrm{L}^{-1}\right)$ & $78,5-195,5$ & 137,0 & $127,1-175,4$ & 151,5 \\
\hline Ammonium $\left(\mu \mathrm{g} \cdot \mathrm{L}^{-1}\right)$ & $40,1-74,6$ & 57,4 & $218,2-349,2$ & 283,7 \\
\hline Azote total $\left(\mathrm{mg} \cdot \mathrm{L}^{-1}\right)$ & $1,7-2,6$ & 2,2 & $2,6-3,8$ & 3,2 \\
\hline NT/PT & $114,7-342,8$ & 228,8 & $118,9-204,8$ & 161,8 \\
\hline Matière organique $\left(\mathrm{mg} \cdot \mathrm{L}^{-1}\right)$ & $4,4-8,6$ & 6,5 & $6,2-7,6$ & 6,9 \\
\hline
\end{tabular}




\subsection{Dynamique du phytoplancton}

Parmi les 69 taxons phytoplanctoniques inventoriés dans le lac Oued Mellah, la majorité des taxons appartient aux Chlorophycées $(36,76 \%)$, aux Cyanobactéries $(26,47 \%)$ et aux Diatomées $(19,11 \%)$. Les Chrysophycées sont représentées uniquement par l'Haptophycée Prymnesium parvum. Malgré sa richesse spécifique relativement importante, le peuplement phytoplanctonique est largement dominé sur le plan quantitatif par quelques espèces seulement. Ce sont surtout des espèces potentiellement toxiques telles que les Cyanobactéries Microcystis ichthyoblabe, Anabaena sp. et Oscillatoria sp. et l'Haptophycée $P$. parvum qui dominent et peuvent occasionner des blooms particulièrement à la fin printemps, été ou au début automne (figures $2 A, 2 B$ ). $P$. parvum, inventoriée pour la première fois dans les lacs du Maroc, occasionne des blooms surtout en période automnale. Contrairement aux Cyanobactéries, cette espèce d'Haptophycée ne forme pas d'écumes flottantes mais elle communique à l'eau une coloration jaune dorée d'où l'appellation de marées jaunes donnée à ce type d'efflorescence algale. En 1998, $P$. parvum, apparaît après une longue période (février-octobre) de dominance des cyanobactéries du genre Oscillatoria. Les biomasses maximales produites par $P$. parvum en novembre et décembre 1998 sont respectivement de l'ordre de 38 et $40 \mathrm{mg}$ poids frais. $L^{-1}$ représentant plus de $80 \%$ de la biomasse phytoplanctonique totale. L'année 1999 est marquée par le développement d'un bloom important à $M$. ichthyoblabe à la fin du printemps avec une biomasse maximale de $298 \mathrm{mg}$ poids frais $\mathrm{L}^{-1}$. Dès la fin de l'été et parallèlement au déclin du bloom à Anabaena sp., $P$. parvum commence sa prolifération et forme un bloom en septembre et octobre correspondant à des biomasses maximales de $45 \mathrm{mg}$ poids frais $\mathrm{L}^{-1}$. Bien que les biomasses produites soient légèrement plus élevées par rapport à l'année 1998, l'importance relative de $P$. parvum est plus faible puisqu'elle ne constitue que $34 \%$ (septembre) à $66 \%$ (octobre) de la biomasse phytoplanctonique totale. Ces faibles pourcentages sont dus au développement du bloom à Anabaena sp. qui représente de 56 à $88 \%$ de la biomasse durant la fin de l'été.

L'évolution saisonnière de la chlorophylle a dans le lac montre que les plus fortes concentrations sont mesurées en novembre-décembre 1998 et septembre-octobre 1999, périodes de prolifération maximale des blooms à Prymnesium parvum (figure $2 C$ ). Les pics de chlorophylle a enregistrés $\left(257 \mu \mathrm{g} \cdot \mathrm{L}^{-1}\right.$ en décembre 1998 et $322 \mu \mathrm{g} \cdot \mathrm{L}^{-1}$ en septembre 1999) correspondent également aux valeurs maximales annuelles. Le pic enregistré en mai $1999\left(222 \mu \mathrm{g} \cdot \mathrm{L}^{-1}\right)$ correspond au développement du bloom à Microcystis ichthyoblabe. L'évolution de la chlorophylle a ne reflète pas toujours celle de la biomasse phytoplanctonique estimée à partir des dénombrements cellulaires. En effet, les valeurs du rapport chlorophylle a/Poids frais varient de 0,69\% en décembre 1998 (dominance de $P$. parvum à $98 \%$ ), à $0,07 \%$ en mai 1999 (dominance de $M$. ichthyoblabe à $99 \%$ ) et à 0,25\% en septembre 1999 (codominance de Anabaena sp. à $57 \%$ et $P$. parvum à $34 \%$ ). Ces variations peuvent s'expliquer par la nature et la taille des espèces dominantes, par leur état physiologique ainsi que par l'influence des conditions mésologiques (température, lumière et nutriments).

\subsection{Dynamique de Prymnesium parvum}

Le suivi rapproché in situ du développement de $P$. parvum en surface du lac durant les années 1998 et 1999 permet d'étudier sa cinétique de croissance 


\section{A Biomasse (mg poids frais. $\left.L^{-1}\right)$}

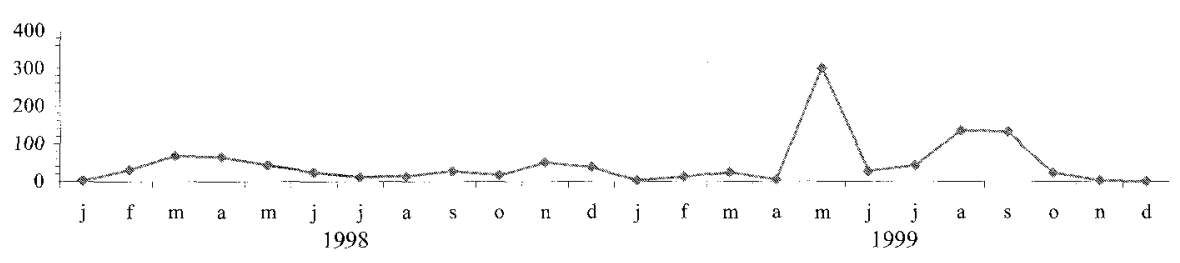

$\begin{array}{lll}\text { B Biomasses relatives (\% poids frais) } & \text { DCyano. } & \text { DChryso. } \\ \text { Bacillario. } & \text { Dautres }\end{array}$
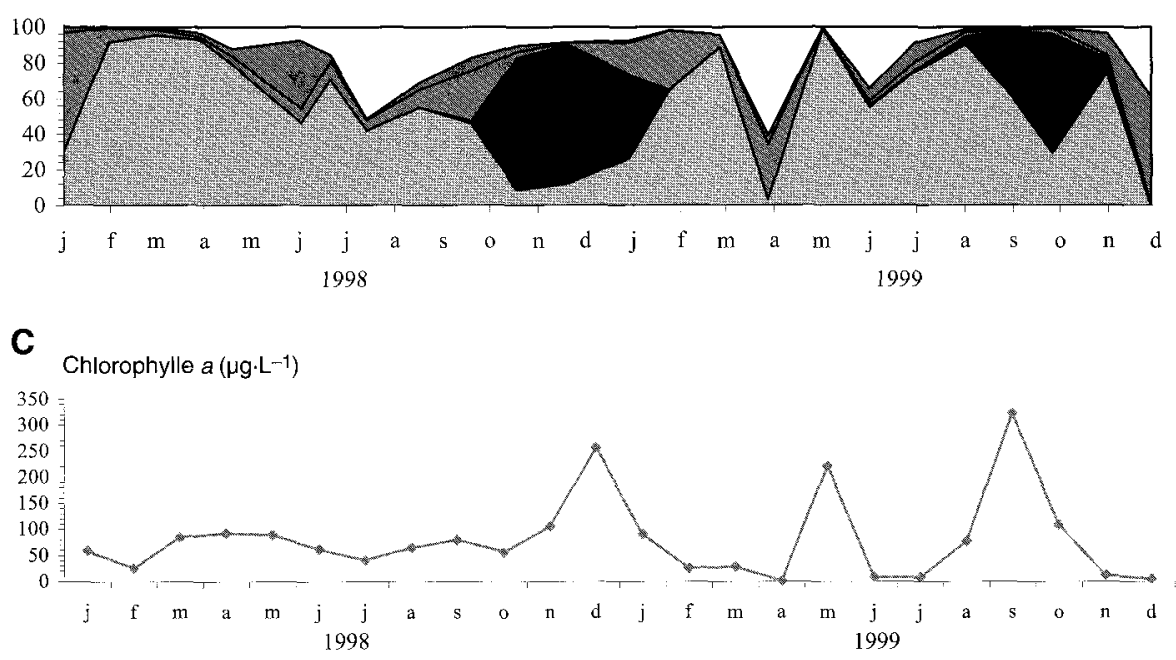

Figure 2 Évolution de la biomasse phytoplanctonique totale (mg poids frais $\left.\cdot L^{-1}\right)(A)$, des biomasses relatives (\% poids frais) $(B)$ et de la chlorophylle a $\left(\mu \mathrm{g} \cdot \mathrm{L}^{-1}\right)(\mathrm{C})$ dans les eaux de surface du lac.

Evolution of total phytoplanktonic biomass (mg fresh weight. $\left.L^{-1}\right)(A)$, relative biomass (\% fresh weight) $(B)$ and chlorophyll a $\left(\mu \mathrm{g} \cdot L^{-1}\right)(C)$ in water surface.

(figure 3). Cette dernière présente la même allure avec trois phases distinctes : la phase de croissance exponentielle, la phase stationnaire et la phase de déclin. Cependant, le mode de croissance diffère d'une année à l'autre par la période de prolifération et par l'importance de la biomasse produite. En 1998, la phase de croissance débute début octobre avec des faibles biomasses et s'étend jusqu'à mi-novembre ou la densité cellulaire atteint $135 \cdot 10^{6} \mathrm{cell} \cdot \mathrm{L}^{-1}$. Le taux maximal de croissance est de $0,64 \mathrm{j}^{-1}$. La période de mi-novembre à midécembre correspond à la phase stationnaire avec des densités moyennes de $140 \pm 5 \cdot 10^{6}$ cell. $L^{-1}$ et un taux moyen de croissance nul. Au-delà de cette période, les densités de $P$. parvum subissent une baisse brutale. En 1999, $P$. parvum démarre sa croissance plutôt comparativement à l'année 1998. La phase de croissance s'étale du début août à fin septembre avec un taux maximal de croissance de $0,64 \mathrm{j}^{-1}$. Au cours de la période du 29 septembre au 10 octobre correspondant à la phase stationnaire, $P$. parvum atteint des densi- 


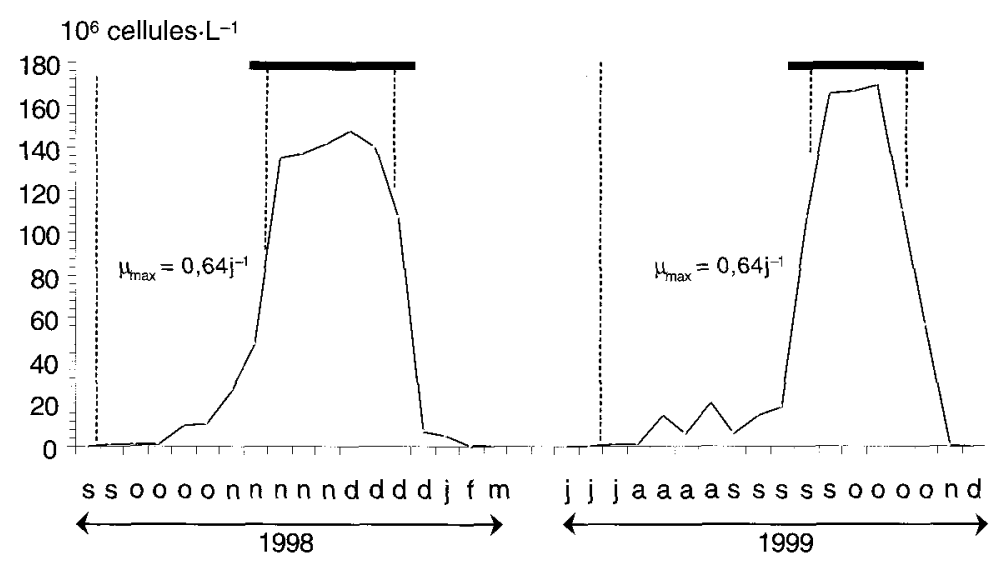

Figure 3 Cinétique de croissance de Prymnesium parvum $\left(\times 10^{6} \mathrm{cell} \cdot \mathrm{L}^{-1}\right)$ dans les eaux de surface.

$\mu$ : taux de croissance $\left(j^{-1}\right) ;(-)$ : Périodes de mortalité des poissons.

Growth of Prymnesium parvum $\left(\times 10^{6}\right.$ cell. $\left.L^{-1}\right)$ in water surface.

$\mu$ : growth rate $\left(\zeta^{-1}\right)$; ( - Fish mortality periods.

tés légèrement supérieures $\left(167 \pm 2 \cdot 10^{6}\right.$ cell $\left.\cdot L^{-1}\right)$ en comparaison avec celles de la même phase de l'année 1998. À partir du 10 octobre, $P$. parvum commence son déclin progressif pour disparaître totalement en novembre.

L'analyse de la distribution verticale de $P$. parvum au cours des périodes de bloom montre que cette espèce colonise toute la colonne d'eau (figure 4). En surface, la biomasse est légèrement plus faible comparativement à celle mesurée à $1 \mathrm{~m}$ de profondeur où la chrysophycée semble trouver des conditions de croissance optimale. Au-delà de cette profondeur, on note une réduction de la biomasse de Prymnesium avec une distribution verticale plus ou moins homogène.

Prymnesium (mg poids frais. $L^{-1}$ )

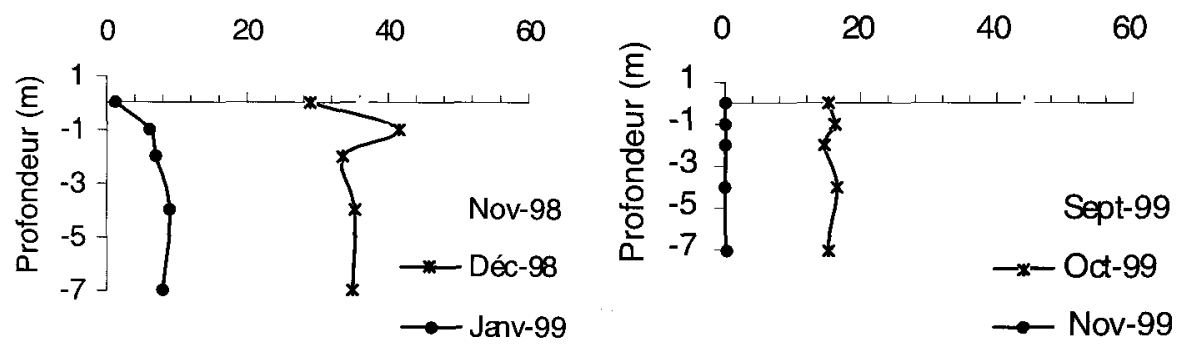

Figure 4 Profils verticaux de Prymnesium parvum (mg poids frais $\cdot \mathrm{L}^{-1}$ ) en période de blooms.

Vertical distribution of Prymnesium parvum (mg fresh weight $\cdot L^{-1}$ ) during bloom periods. 


\subsection{Mortalités des poissons}

La figure 3 montre que la période de mortalité des poissons coïncide chaque année avec le développement maximal des blooms à $P$. parvum en fin de phase de croissance et pendant la phase stationnaire. Ce résultat laisse supposer l'implication plus que probable de $P$. parvum dans ces mortalités. L'examen des profils verticaux de la saturation en oxygène (figure 5) montre que les valeurs en période de blooms à $P$. parvum dépassent, dans tous les cas, $58 \%$, ce qui écarte l'hypothèse de mortalité par simple anoxie. D'autre part, l'examen histopathologique de quelques poissons intoxiqués montre que les branchies sont les organes les plus endommagés avec des hémorragies nettes et une importante destruction du tissu branchial. Ces symptômes ne correspondent pas à ceux souvent signalés dans le cas de mortalité par asphyxie. Pratiquement toutes les espèces de poissons (carpe, barbeau, anguille, perche soleil, gambusie) présentes dans le lac ont été intoxiquées. Cependant, la carpe, le barbeau et la perche soleil restent les espèces les plus touchées. Les mortalités touchent également d'autres animaux aquatiques tels que les crevettes, les mollusques bivalves et d'autres invertébrés benthiques. L'estimation de la quantité de poissons intoxiqués n'est pas facile à réaliser à cause de la distribution très hétérogène des poissons morts tout au long des berges du lac.

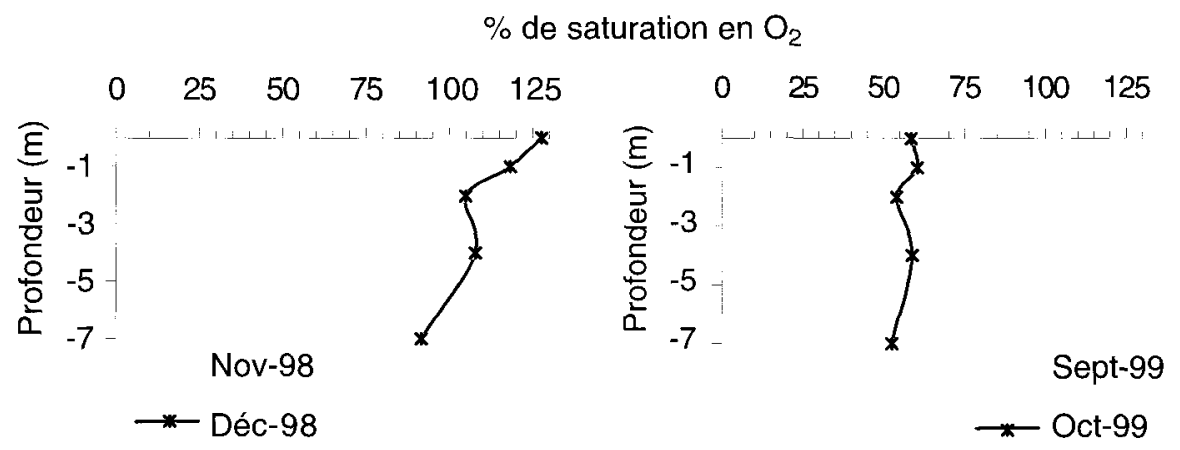

Figure 5 Profils verticaux des teneurs en oxygène dissous (\%) en période des blooms à Prymnesium parvum.

Vertical distribution of dissolved oxygen (\%) during Prymnesium parvum blooms.

\section{4 - DISCUSSION}

Parmi les dix espèces décrites du genre Prymnesium (GREEN et LEADBEATER, 1994), P. parvum, $P$. patelliferum, $P$. saltans et $P$. calathiferum ont été signalés comme responsables de blooms ichthyotoxiques dans divers régions du monde (voir les synthèses d'EDVARDSEN et PAASCHE (1998) et de MOESTRUP (1994)). À l'exception du bloom à $P$. calathiferum signalé dans la baie Bream dont les eaux sont purement marines (34-35 \%) (CHANG, 1985), les blooms à Prymnesium sont fréquemment notés dans les eaux saumâtres à faible salinité (1-12 \%o). Ces blooms se développent souvent dans des lacs peu profonds, des étangs ou des 
lagunes à surface limitée (EDVARDSEN et PAASCHE, 1998). Le développement maximal des blooms à $P$. parvum dans le lac peu profond de Oued Mellah coïncide avec des salinités entre 8 et 12,6\%. Ces résultats sont en parfait accord avec les travaux d'OTTERSTRØM et STEEMAN NIELSEN (1940) qui rapportent des blooms intenses de $P$. parvum à une salinité de $8 \%$ et avec ceux de COMIN et FERRER (1978) sur une lagune du delta de l'Ebre qui signalent le développement du bloom à une salinité de 11,6\%. Cependant, des blooms à $P$. parvum ont été signalés récemment dans des lacs saumâtres moins salés tels le lac Massaciuccoli en Italie (1,3-3,6\%) (MATTIOLI et SIMONI, 1999) et le lac Vargsundet à Aland en Finlande (2,22 \%) (LINDHOLM et al., 1999). L'ensemble de ces données montrent que malgré sa large distribution dans les eaux saumâtres, $P$. parvum est une espèce euryhaline. Dans le lac Oued Mellah la période de croissance de $P$. parvum se situe chaque année en fin de l'été et en automne et coïncide avec des températures comprises entre $15^{\circ}$ et $24^{\circ} \mathrm{C}$. Cependant, le développement maximal semble être favorisé par des températures comprises entre $20^{\circ}$ et $24^{\circ} \mathrm{C}$. Ces résultats sont en accord avec les données de la littérature. Ainsi, LINDHOLM et al. (1999) a constaté, sur le lac Vargsundet, un bloom à $P$. parvum associé à des températures entre $20^{\circ}$ et $25^{\circ} \mathrm{C}$. Toutefois, LARSEN et BRYANT (1998) démontrent avec différentes souches de $P$. parvum isolées au laboratoire que le taux maximal de croissance est obtenu à une température de $15^{\circ} \mathrm{C}$ mais que ces souches peuvent croître à des températures comprises entre $5^{\circ}$ et $30^{\circ} \mathrm{C}$. En définitive, $P$. parvum semble tolérer une large gamme de températures et former des blooms entre $5^{\circ} \mathrm{C}$ (KRASCHNOCHEK et ABRAMOVICH, 1971 ; MATTIOLI et SIMONI, 1999) et $30^{\circ} \mathrm{C}$ (SHILO, 1972). En période de développement maximal de $P$. parvum, la luminosité incidente présente des valeurs moyennes de $1238 \mu \mathrm{mol} \cdot \mathrm{m}^{-2} \cdot \mathrm{s}^{-1}$ en 1998 et de $1304 \mu \mathrm{mol} \cdot \mathrm{m}^{-2} \cdot \mathrm{s}^{-1}$ en 1999 . Ces valeurs sont beaucoup plus élevées que les intensités optimales de croissance (65$\left.200 \mu \mathrm{mol} \cdot \mathrm{m}^{-2} \cdot \mathrm{s}^{-1}\right)$ données pour plusieurs souches de $P$. parvum et $P$. patelliferum (LARSEN et BRYANT, 1998). P. parvum se développe dans le lac Oued Mellah en présence de concentrations limitantes en phosphore (tableau 2). Des résultats similaires ont été signalés par AURE et FRANCISCO (1991) et LINDHOLM et al. (1999). La prolifération des blooms à $P$. parvum malgré la déficience aiguë en nutriments, notamment en orthophosphates, laisse suggérer l'intervention de propriétés physiologiques adaptatives à ces conditions. En effet, $P$. parvum dispose d'une phosphatase alcaline extracellulaire qui permet à l'algue d'utiliser le phosphore organique présent dans son environnement immédiat (WYNNE et RHEE, 1988). En période de blooms à $P$. parvum, les teneurs en azote semblent également limitantes, particulièrement en nitrates (tableau 2). $P$. parvum peut utiliser en plus des nitrates et de l'ammonium, des formes organiques (acides aminés tels que la méthionine et l'éthionine) comme source d'azote (RAHAT et HOCHBERG, 1971). En effet, cette espèce possède au niveau de la surface cellulaire des L-amino acides oxydases qui oxydent les acides aminés, en produisant de l'ammonium utilisé par la cellule (PALENIK et MOREL, 1990). Le rapport NT/PT est élevé (115-343) et semble favoriser le développement des blooms à $P$. parvum dans le lac Oued Mellah comme dans d'autre plans d'eau (AURE et FRANCISCO 1991 ; LINDHOLM et al., 1999). L'hypothèse sur l'utilisation de sources organiques pour faire face au déficit en nutriments est compatible avec les teneurs relativement élevées en matières organiques dissoutes $(4,4$ $8,6 \mathrm{mg} \cdot \mathrm{L}^{-1}$ ) en période de prolifération de blooms à $P$. parvum. Bien que les travaux sur les capacités hétérotrophes de Prymnesium restent limités, certains auteurs ont tenté d'expliquer la relation entre le développement de cette espèce 
et la charge organique. RAHAT et JAHN (1965) ont montré que cette espèce peut se développer à l'obscurité prolongée dans un milieu de culture enrichi en glycérol et en serine. En plus de l'osmotrophie, la phagotrophie a été également démontrée chez $P$. parvum (NYGAARD et TOBIESEN, 1993) et chez $P$. patelliferum (TILLMANN, 1998). Ce caractère mixotrophe constitue un avantage compétitif pour Prymnesium par rapport aux autres espèces strictement hétérotrophes ou strictement photoautotrophes et constitue une source d'acquisition supplémentaire d'éléments indispensables tels que $\mathrm{C}, \mathrm{N}$ et $\mathrm{P}$ sous des conditions de déficit en éléments minéraux (GRANÉLI et al., 1999).

Au cours des deux périodes de bloom en 1998 et 1999, P. parvum est l'espèce dominante du phytoplancton (34 à plus de $80 \%$ de la biomasse totale) et son développement succède au déclin des blooms à Cyanobactéries (Oscillatoria sp. en 1998 et Anabaena sp. en 1999). MATTIOLI et SIMONI (1999) signalent que les blooms à $P$. parvum précèdent les blooms à Cyanobactéries (Lyngbya $s p$. et Microcystis sp.) dans le lac Massaciuccoli en Italie. LINDHOLM et al. (1999) rapportent deux blooms simultanés de $P$. parvum (dans l'épilimnion) et Planktothrix agardhii (dans le métalimnion) dans le lac Vargsundet en Finlande. Dans le même lac, JOHNSEN et LEIN (1989) ont rapporté que les filaments de Planktothrix pouvaient être colonisés par Prymnesium. L'ensemble des ces observations ne permet pas de conclure sur la nature des interactions entre les blooms à Cyanobactéries et ceux à Prymnesiophycées.

Les fortes mortalités de poissons enregistrées dans le lac Oued Mellah en 1998 et 1999 coïncident avec la fin de la phase de croissance exponentielle et la phase stationnaire du développement des blooms à $P$. parvum. Sur des souches de Prymnesium produites en culture, MOESTRUP (1994) et SHILO (1967) rapportent que la toxicité est surtout exprimée en phase de croissance et en phase stationnaire. La parfaite coïncidence entre ces blooms et les mortalités de poissons permet la mise en cause de cette espèce. De même, la relative abondance des données de la littérature sur la toxicité des Haptophycées (tableau 3) laisse également présager que $P$. parvum peut être la cause des mortalités observées en l'absence de toute autre cause évidente dans le lac.

Depuis que $P$. parvum a été reconnue comme ichthyotoxique, cette microalgue et ses toxines ont été amplement étudiées (LARSEN et BRYANT, 1998). Les exotoxines produites par ce phytoflagellé contiennent des composés capables d'induire des réponses physiologiques variées, telles que l'ichthyotoxicité, la cytotoxicité, la neurotoxicité et l'activité antibactérienne (IGARASHI et al., 1996, 1999 ; MELDAHL et FONNUM, 1993 ; SHILO, 1971). L'ichthyotoxine puissante découverte par REICH et ASCHNER (1947) chez $P$. parvum est nommée prymnesine (YARIV et HESTRIN, 1961). Ce n'est que récemment que IGARASHI et al. $(1996,1998,1999)$ ont pu isoler et identifier les composés ichthyotoxiques et hémolytiques, prymnesine-1 et prymnesine-2, à partir des cultures de $P$. parvum. Tous les animaux aquatiques possédant des branchies ou un stade de vie branchial sont sensibles aux biotoxines produites par Prymnesium (PREMAZZI et VOLTERRA, 1993).

Il a été démontré également que la toxicité de $P$. parvum était fortement liée à certains facteurs environnementaux. En effet, elle augmente considérablement en conditions de carence en phosphore (RAHAT et SPIRA, 1967 ; SHILO, 1971), lors d'une limitation en phosphore et une forte saturation en oxygène (IGARASHI et al., 1995 ; MELDAHL et FONNUM, 1993 ; SHILO, 1981) et avec un 
Tableau 3 Événements toxiques d'Haptophycées.

Table 3 Haptophycean toxic events.

\begin{tabular}{|c|c|c|c|c|}
\hline Années & Localités & Espèces & $\begin{array}{l}\text { Animaux } \\
\text { affectés }\end{array}$ & Réf. \\
\hline $1900-1933$ & $\begin{array}{l}\text { Lacs Waterneverstorfer, } \\
\text { Allemagne }\end{array}$ & Prymnesium parvum & Poissons & 1 \\
\hline 1920 & Lac Workum, Hollande & Prymnesium parvum & Poissons & 2 \\
\hline 1970 & Darb, Allemagne & Prymnesium parvum & Poissons & 3 \\
\hline $1972-1977$ & Lac Massaciuccoli, Italie & Prymnesium sp. & Poissons & 4 \\
\hline 1975 & $\begin{array}{l}\text { Étang peu profond } \\
\text { de carpiculture, Fehrmann, } \\
\text { Allemagne }\end{array}$ & Prymnesium parvum & Poissons & 5 \\
\hline 1988 & $\begin{array}{l}\text { Skagerrak Kattegat, Sound, } \\
\text { Mer Baltique }\end{array}$ & $\begin{array}{l}\text { Chrysochromulina } \\
\text { plylepis }\end{array}$ & $\begin{array}{l}\text { Effets à grande } \\
\text { échelle }\end{array}$ & 6 \\
\hline $1989-1996$ & $\begin{array}{l}\text { Fermes piscicoles, système } \\
\text { Sandsfjord, Norvège }\end{array}$ & $\begin{array}{l}\text { Prymnesium parvum et } \\
\text { Prymnesium patelliferum }\end{array}$ & Poissons & 7,8 \\
\hline 1990 & Rügen, Allemagne & Prymnesium saltans & Poissons & 9 \\
\hline $1990-1992$ & Petite crique, Finlande & Prymnesium parvum & Poissons & 10 \\
\hline 1991 & $\begin{array}{l}\text { Lac peu profond côtier, } \\
\text { Kyrkjärden, Suède }\end{array}$ & Prymnesium parvum & Oiseaux de mer & 11 \\
\hline 1997 & $\begin{array}{l}\text { Lac de Vargsundet, Aland, } \\
\text { Finlande }\end{array}$ & Prymnesium parvum & $\begin{array}{l}\text { Poissons + } \\
\text { mouettes } \\
+ \text { allergie chez } \\
\text { des baigneurs }\end{array}$ & 12 \\
\hline 1999 & Lac Massaciuccoli, Italie & Prymnesium patelliferum & Poissons & 13 \\
\hline $1998-1999$ & Lac Oued Mellah, Maroc & Prymnesium parvum & $\begin{array}{l}\text { Poissons + } \\
\text { quelques } \\
\text { invertébrés } \\
\text { aquatiques }\end{array}$ & $\begin{array}{l}\text { Présent } \\
\text { travail }\end{array}$ \\
\hline
\end{tabular}

$1:$ OTterstrøm et STEEMAN NIELSEN $(1940) ; 2:$ LIEBERT et DEERNS $(1920) ; 3:$ KalbE et TSCHEUSCHLÜTER (1972) ; 4 : SIMONI (1977) ; 5 : HICKEL (1976) ; 6 : AKSNES et al. (1989) ; 7 : EIKEN et THRODSEN $(1993) ; 8:$ EDVARDSEN et PAASCHE $(1998) ; 9 ;$ KELL et NOACK $(1991) ; 10:$ LINDHOLM et VIRTANEN (1992); 11 : HOLMQUIST et WILLÉN (1993) ; 12 : LINDHOLM et al. (1999) ; 13 : MATTIOLI et SIMONI (1999).

${ }^{*}$ ) Après plusieurs investigations sur la physiologie et la génétique de $P$. parvum et $P$. patelliferum, LARSEN (1999) propose le regroupement des deux espèces en une seule avec la nouvelle nomination Prymnesium parvum f. patelliferum (GREEN, HIBBERD et PIENAAR) A. Larsen stat. Nov. pour $P$. patelliferum.

rapport N/P élevé (AURE et FRANCISCO, 1992). Ces trois conditions sont réunies dans le lac Oued Mellah. Par ailleurs, les résultats des travaux sur l'effet de la salinité, la température et la lumière sur la toxicité de $P$. parvum restent souvent contradictoires (EDVARDSEN et al., 1996 ; LARSEN et BRYANT, 1998). 


\section{5 - CONCLUSION}

Le présent travail constitue une première contribution à l'étude des blooms à $P$. parvum dans le lac Oued Mellah et apporte des éléments d'informations sur son éventuelle implication dans les mortalités de poissons. II apporte des données sur les conditions écologiques particulières favorisant l'installation et la prolifération des « marées jaunes " en zone semi-aride. Des travaux sur l'autoécologie, l'écophysiologie et la toxicité de $P$. parvum isolée et cultivée au laboratoire sont envisagés pour mieux comprendre le phénomène de marées jaunes et confirmer la mise en cause des blooms à Haptophycées dans la mortalité régulière des poissons dans le lac.

La dégradation de la qualité de l'eau durant la période de dominance de $P$. parvum et les mortalités massives de poissons observées démontrent que la structure et le fonctionnement de la chaîne trophique du lac sont fortement perturbées. Les dégâts causés par $P$. parvum ne se limitent pas uniquement à la perte économique et aux risques écologiques et sanitaires. En raison de la couleur indésirable de l'eau (marée jaune), aux cadavres de poissons morts sur les berges et leur odeur désagréable en décomposition, le lac n'est plus praticable pour divers activités récréatives (loisirs, randonnées, pêche sportive, voile...).

\section{REMERCIEMENTS}

Ce travail a été supporté par le projet PARS N 189 Biologie "Programme d'appui à la recherche scientifique au Maroc ». Nous remercions Mme M. ElAndaloussi Dadi du Laboratoire d'histopathologie Tensift (Marrakech) pour la réalisation de l'étude histopathologique de poissons.

\section{RÉFÉRENCES BIBLIOGRAPHIQUES}

AFNOR, 1994. Recueil des normes françaises, eaux, méthodes d'essais. Ed. Afnor, Paris.

AKSNES D.L., AURE J., FURNES G.K., SKJOLDAL H.R., SAETRE R., 1989. Analysis of the Chrysochromulina polylepis bloom in the Skagerrak, May 1988. Environnemental conditions and possible causes. Bergen Sci. Center Publ. BSC 89/1.

AURE J., FRANCISCO R., 1991. Oceanographic conditions in the Sandsfjord sys- tem, western Norway, after a bloom of the toxic Prymnesiophyte Prymnesium parvum Carter in August 1990. Sarsia, $76,247-254$.

BELL M.G.W., 1980. Environmental pressures on inland waters. The Norfolk Broads. Wat. Sci. Technol. Prog., 13, 249-264.

CAPBLANCQ J., 1982. Phytoplancton et production primaire. In : Écologie du plancton des eaux continentales, Coll. Écologie, Masson, Paris, 16, 198 p. 
CARTER N., 1937. New or interesting algae from brackish water. Archiv Protistenk, 90, 1-68.

CHANG F.H., 1985. Preliminary toxicity test of Prymnesium calathiferum n.sp. isolated from New Zealand. In: ANDERSON D.M., WHITE A.W., BADEN D.G. (eds.), Toxic Dinoflagellates, Elsevier, New York, 109-112.

CHRETIENNOT-DINET M.-J., 1990. Atlas du phytoplancton marin, V. III, eds. CNRS, Paris, $120 \mathrm{p}$.

COMIN F.A., FERRER X., 1978. Desarrollo masivo del fitoflagelado Prymnesium parvum Carter (Haptophyceae) en una laguna costera del delta Ebro. Oecol. Aquat., 3, 207-210.

DERRAZ M., 1995. Étude de l'eutrophisation de la retenue du barrage El Kansera (Maroc). Th. Doct. Univ. Moulay Ismail, Méknès, $120 \mathrm{p}$.

EDVARDSEN B., LARSEN A., KRISTIANSEN S., PAASCHE E., 1996. The effect of growth stage, salinity, temperature and nutrient limitation on the toxicity of Chrysochromulina polylepis (Haptophyceae). In: EDVARDSEN B. Toxicity, autoecology and life history of Chrysochromulina polylepis (Haptophyceae), Th. Doct., Univ. Oslo, Nonway, $160 \mathrm{p}$.

EDVARDSEN B., PAASCHE E., 1998. Bloom dynamics and physiology of Prymnesium and Chrysochromulina. In: ABDERSON, D.M., CEMBELLA A.D., HALLEGRAEFF G.M. (eds.), The physiological ecology of harmful algal blooms, Springer-Verlag, Heidelberg, 193-208.

EIKREM W., THRONDSEN J., 1993. Toxic prymnesiophytes identified from Norwegian coastal waters. In: SMAYDA T.J., SHIMIZU Y. (eds.), Toxic phytoplankton blooms in the sea, Elsevier Science Publishers,687-692.

FARROW G.A., 1969. Note on the association of Prymnesium with fish mortalities. Wat. Res., 3, 375-379.

GRANÉLI E., CARLSSON P., LEGRAND E., 1999. The role of $C, N$ and $P$ in dissolved and particulate organic matter as a nutrient source for phytoplankton growth, including toxic species. Aquat. Ecol., 33, 17-27.

GREEN J.C., LEADBEATER B.S.C., 1994. The haptophyte algae. Systematics
Association, Special volume $n^{\circ} 51$, Oxford University Press, New York, $446 \mathrm{p}$.

GUO M., HARRISON P.J., TAYLOR F.J.R., 1996. Fish kills related to Prymnesium parvum N. Carter (Haptophyta) in the People's Republic of China. J. Appl. Phycol., 8, 11-117.

HICKEL B., 1976. Fischsterben in einem Karpfenteich bei einer Massenentwicklung des toxschen Phytoflagellaten Prymnesium parvum Carter (Haptophyceae). Arch. Fisch. Wiss., 27, 143-148.

HOLMQUIST W., WILLÉN T., 1993. Fish mortality caused by Prymnesium parvum? Vatten, 49, 110-115.

IGARASHI T., ARITAKE S., YASUMOTO T., 1998. Biological activities of Prymnesin2: isolated from the red tide alga Prymnesium parvum. Nat. Toxins; 6, 35-41.

IGARASHI T., SATAKE M., YASUMOTO T., 1996. Prymnesin-2: a potent ichthyotoxic and hemolytic glycoside isolated from the red tide alga Prymnesium parvum. J. Am. Chem. Soc., 118, 479-480.

IGARASHI T., SATAKE M., YASUMOTO T., 1999. Structures and partial stereo chemical assignments for Prymnesin- 1 and Prymnesin-2: Potent hemolytic and ichthyotoxic glycosides isolated from the red tide alga Prymnesium parvum. J. Am. Chem. Soc., 121, 8499-8511.

JOHNSEN T.M., LEIN T.E., 1989. Prymnesium parvum Carter (Prymnesiophyceae) in association with macroalgae in Ryfylke, Southwestern Norway. Sarsia, 74, 277-281.

KALBE L., TSCHEU-SCHLÜTER M., 1972. Fischsterben durch Prymnesium parvum Carter in einem Nebengewässer eines Boddens der Ostsee. Z. Binnenfischerei DDR, 19, 40-44.

KELL V., NOACK B., 1991. Fischsterben durch Prymnesium saltans Massart im Kleinen Jasmunder Bodden (Rügen) im April 1990. J. Appl. Ichthyol., 7, 187-192.

KONONEN K., SIVONEN K., LEHRIMAKI J., 1993. Toxicity of phytoplankton blooms in the gulf of Finland and gulf of Bothnia, Baltic sea. In: SMAYDA T.J., SHIMIZU Y. (eds.), Toxic phytoplankton blooms in the sea, Elsevier Science Publishers, 269-274.

KRASNOSHCHEK G.P., ABRAMOVICH L.S., 1971. Mass development of Prymnesium 
parvum Cart. in fish breading ponds. Hydrobiol. J., 7, 54-55.

LARSEN A., 1999. Prymnesium parvum and $P$. patelliferum (Haptophyta) - one species. Phycologia, 38, 541-543.

LARSEN A., BRYANT S., 1998. Growth rate and toxicity of Prymnesium parvum and Prymnesium patelliferum (Haptophyta) in response to changes in salinity, light and temperature. Sarsia, 83, 409-418.

LIEBERT F., DEERNS W.M., 1920. Onderzoek nach oorzaak van een vischstrefte in den polder Workumer - Niewland, nabiji Workum - Rijksintituut voor Visscherijonderzoek. Verhandelinger en rapporten, 81-93.

LINDHOLM T., ÖHMAN P., KURKIHELASMO K., KINCAID B., MERILUOTO J., 1999. Toxic algae and fish mortality in a brackish-water lake in Åland, SW Finland. Hydrobiologia, 397, 109-120.

LINDHOLM T., VIRTANEN T., 1992. A bloom of Prymnesium parvum in a small coastal inlet in Dragsfjärd southwestern Finland. Env. Toxicol. Wat. Qual., 7, 165-170.

LORENZEN C.J., 1967. Determination of chlorophyll and pheo-pigments: spectrophotometric equations. Limnol. Oceanogr., 12, 343-346.

LOUDIKI M., OUDRA B., SABOUR B., SBIYYAA B. FRANCA S., VASCONCELOS V. 2000. Geographic distribution and taxonomy of potential cyanobacterial strains in Morocco. $9^{\text {th }}$ International Conference on Harmful Algal Blooms (HAB2000), Tasmania, Australia.

MATTIOLI M., SIMONI F., 1999. Toxicity of Prymnesium in the shallow lake of Massaciuccoli (Migliarino-San RossoreMassaciuccoli Regional Park - Pisa Tuscany). IOC Newsletter, Toxic Algae and algal blooms, 19, 7 .

MELDAHL A.S., FONNUM F., 1993. The effect of toxins of Prymnesium patelliferum on neurotransmitter transport mechanisms. The development of a sensitive test method. J. Tox. Env. Health, 38, 57-67.

MOESTRUP O., 1994. Economic aspects: blooms, nuisance species and toxins. In: GREEN J.C., LEADBEATER J.C., LEADBEATER B.S.C. (eds.), The Haptophyte Algae, Oxford University Press, New York, 265-285.
NYGAARD K., TOBIESEN A., 1993. Bacterivory in algae: a survival strategy during nutrient limitation. Limnol. Oceanogr., 38, 273-279.

OTTERSTRØM C.V., STEEMAN NIELSEN E., 1940. Two cases of extensive mortality in fishes caused by flagellate Prymnesium parvum Carter. Rep. Dan. Biol. Station, 44, 1-24.

OUDRA B., LOUDIKI M., SBIYYAA B., VASCONCELOS V., 1998. Occurrence of hepatotoxic Microcystis aeruginosa water bloom in a eutrophic moroccan lake reservoir. In: REGUERA B., BLANCO J., FERNANDEZ M.L., WYATT T., XUNTA DE GALICIA, IOC-UNESCO (eds), Harmful Algae, 29-31.

PALENIK B., MOREL F.M.M., 1990. Comparison of cell-surface L-amino acid oxidases from several marine phytoplankton. Mar. Ecol. Prog. Ser., 59, 195-201.

PREMAZZI, G., VOLTERRA L., 1993. Brackish toxins - Prymnesium parvum toxins. In: Joint Research Centre (C.E.C) (eds.), Microphyte toxins: A manual for toxin detection, environmental monitoring and therapies to counteract intoxications, 123-128.

RAHAT M., HOCHBERG A., 1971. Ethionine and methionine metabolism by the chrysomonad flagellate Prymnesium parvum. J. Protozool., 18, 378-382.

RAHAT M., JAHN T.L., 1965. Growth of Prymnesium parvum in the dark; note on ichthyotoxin formation. J. Protozool., 12, 246-250.

RAHAT M., SPIRA G., 1967. Specificity of glyceral for dark growth of Prymnesium parvum. J. Protozool., 14, 45-48.

REICH K., ASCHNER M., 1947. Mass development and control of the phytoflagellate Prymnesium parvum in fishponds in Palestine. Palestinian J. Bot. Jerusalem Ser., 4, 14-23.

RODIER J., 1984. L'analyse de l'eau, $7^{e}$ édition, Dunod, Paris.

SABOUR B., LOUDIKI M., OUDRA B., OUBRAIM S., FAWZI B., FADLAOUI S., CHLAIDA M., 2000. First data on Microcystis ichthyoblabe Kütz. toxic blooms in Morocco: case of Oued Mellah hypereutrophic lake. International Conference on Toxic Cyanobacterial Blooms (ICTCB), Rabat, Morocco. 
SHILO M., 1967. Formation and mode of action of algal toxins. Bacteriol. Rev., 31, 180-193.

SHILO M., 1971. Toxins of Chrysophyceae. In: KADIS S., CIEAGLER A., AJL S.J. (eds.), Microbial toxins, Vol. VII, Academic Press, New York, 67-103.

SHILO M., 1972. Toxigenic algae. In: HOCKENHULL O.J.D. II (ed.), Progress in Industrial Microbiology, Churchill Livingstone Press, Edinburgh, 233-265.

SHILO M., 1981. The toxic principles of Prymnesium parvum. In: CARMICHAEL W.W. (ed.), The water environment. Algal toxins and health, Plenum Press, New York, 37-47.

SIMONI F., 1977. Sulle cause delle morie dei pesci nel lago di Massaciuccoli negli anni 1972-1977. Rivista Haliana d'Igiene, Vol. XXXVII N. 5-6.

TILLMANN U., 1998. Phagotrophy by a plastidic haptophyte, Prymnesium patelliferum. Aquat. Microb. Ecol., 14, 155-160.

UTERMÖHL H., 1958. On the perfecting of quantitative phytoplankton method. Int. Ass. Theor. Appl. Limnol. Commun., 9, 1-38.

WYNNE D., RHEE G.Y., 1988. Changes in alkaline phosphatase activity and phosphate uptake in P-limited phytoplankton induced by light intensity and spectral quality. Hydrobiologia, 160, 1723-1728.

YARIV J., HESTRIN S., 1961. Toxicity of the extracellular phase of Prymnesium parvum cultures. J. Gen. Microbiol., 24, 165-175. 